\title{
Least-squares forecast averaging
}

\author{
Bruce E. Hansen* \\ Department of Economics, University of Wisconsin, 1180 Observatory Drive, Madison, WI 53706, United States
}

\section{A R T I C L E I N F O}

\section{Article history:}

Available online 7 September 2008

\section{JEL classification:}

C52

C53

Keywords:

Mallows

AIC

BIC

BMA

Forecast combination

Model selection

\begin{abstract}
A B S T R A C T
This paper proposes forecast combination based on the method of Mallows Model Averaging (MMA). The method selects forecast weights by minimizing a Mallows criterion. This criterion is an asymptotically unbiased estimate of both the in-sample mean-squared error (MSE) and the out-of-sample one-stepahead mean-squared forecast error (MSFE). Furthermore, the MMA weights are asymptotically meansquare optimal in the absence of time-series dependence. We show how to compute MMA weights in forecasting settings, and investigate the performance of the method in simple but illustrative simulation environments. We find that the MMA forecasts have low MSFE and have much lower maximum regret than other feasible forecasting methods, including equal weighting, BIC selection, weighted BIC, AIC selection, weighted AIC, Bates-Granger combination, predictive least squares, and Granger-Ramanathan combination.
\end{abstract}

(c) 2008 Elsevier B.V. All rights reserved.

\section{Introduction}

Forecast combination has a long history in econometrics. While a broad consensus is that forecast combination improves forecast accuracy, there is no consensus concerning how to form the forecast weights. The most recent literature has focused on two particularly appealing methods-simple averaging and Bayesian averaging. The simple averaging method picks a set of models and then gives them all equal weight for all forecasts. The Bayesian averaging method computes forecast weights as a by-product of Bayesian model averaging (BMA).

This paper introduces a new method appropriate for linear models estimated by least squares. The method is to construct forecast combinations using the weights computed by Mallows Model Averaging (MMA), the weights which minimize the generalized Mallows criterion introduced in Hansen (2007). The Mallows criterion is an estimator of mean-squared error (MSE) and mean-squared forecast error (MSFE), and MMA weights are asymptotically optimal with respect to mean-square loss. We therefore expect MMA combination to produce point forecasts with low MSFE. In the context of linear regressions with iid data, Hansen (2007) introduced the idea of model averaging using the weights which minimize the Mallows criterion, demonstrated that the Mallows criterion is an unbiased estimate of the MSE, and showed that the Mallows averaging estimator is asymptotically

\footnotetext{
* Tel.: +1 608263 3880; fax: +1 6082633876 .

E-mail address: bhansen@ssc.wisc.edu.

URL: http://www.ssc.wisc.edu/ bhansen.
}

optimal in the sense of achieving the best possible MSE. Our contribution in this paper is to propose using these weights for forecast combination, show that the Mallows criterion is asymptotically unbiased for the MSE and MSFE when the observations are a stationary time-series, and demonstrate the relative performance of the method in simulation environments.

As mentioned above, two powerful existing methods for forecast combination are simple averaging and Bayesian averaging. Both have been shown to be extremely versatile and successful in applications, yet neither is inherently satisfying. Simple averaging only makes sense if the class of models under consideration is reasonable. If a terrible model is included in the class of forecasting models, simple averaging will pay the penalty. This induces an inherent arbitrariness, and thus the method is incomplete unless augmented by a description of how the initial class of models is determined, which destroys the inherent simplicity of the method.

On the other hand, the fact that BMA relies on priors (over the class of models and over the parameters in the models) means that this method suffers from the arbitrariness which is inherent in prior specification. Furthermore, the BMA paradigm is inherently misspecified. It is developed under the assumption that the truth is one finite-dimensional parametric model out of a class of models under consideration. The goal is to find the "true" model out of this class. This paradigm and goal is inherently misspecified and misguided, as it is more appropriate to think of models as approximations, and that the "true" model is more complex than any of the models in our explicit class. When we fit models, we balance specification error (bias) against overparameterization (variance). The correct goal is to define the object of interest (such as forecast mean-squared error) and then evaluate methods based 
on this criterion, without assuming that we necessarily have the correct model.

Mallows Model Averaging takes exactly the desired approach. The goal is to obtain the set of weights which minimizes the MSE over the set of feasible forecast combinations. The generalized Mallows criterion is an estimate of the MSE and MSFE, and the weights which minimize this criterion are asymptotically optimal in some settings.

The Mallows criterion for model selection was introduced by Mallows (1973), and is similar to the information criteria of Akaike (1973) and Shibata (1980). The asymptotic optimality of model selection by this class of criterion has been studied by Shibata (1980, 1981, 1983), Li (1987), Banasali (1996), Lee and Karagrigoriou (2001), Ing (2003, 2004, 2007) and Ing and Wei (2003, 2005). Akaike (1979) proposed using the exponentiated AIC as model weights, and this suggestion was picked up and expanded by Buckland et al. (1997) and Burnham and Anderson (2002). Hjort and Claeskens (2003) introduced a general class of frequentist model average estimators, including methods similar to Mallows model averaging.

The Bayesian information criterion was introduced by Schwarz (1978) as a method for model selection. There is a large literature on Bayesian Model Averaging; see the review by Hoeting et al. (1999). Some applications in econometrics include Sala-i-Martin et al. (2004), Brock and Durlauf (2001), Avramov (2002), Fernandez et al. (2001a,b), Garratt et al. (2003) and Brock et al. (2003).

The idea of forecast combination was introduced by Bates and Granger (1969), extended by Granger and Ramanathan (1984), and spawned a large literature. Some excellent reviews include Granger (1989), Clemen (1989), Diebold and Lopez (1996), Hendry and Clements (2002), Timmermann (2006) and Stock and Watson (2006). The idea of using Bayesian model averaging for forecast combination was pioneered by Min and Zellner (1993) and its usefulness recently demonstrated by Wright (2003a,b). Stock and Watson $(1999,2004,2005)$ have provided detailed empirical evidence demonstrating the gains in forecast accuracy through forecast combination, and in particular have demonstrated the success of simple averaging (equal weights) along with Bayesian model averaging.

The plan of the paper is simple. Section 2 introduces the model and approximating linear forecasting models. Section 3 introduces forecast combination and reviews existing combination methods. Section 4 presents the Mallows criterion and the MMA forecast combination. Section 5 shows that the Mallows criterion is an asymptotically unbiased estimate of the in-sample mean-squared error. Section 6 shows that this MSE criterion is approximately equivalent to out-of-sample mean-squared forecast error. Section 7 reviews Hansen's (2007) demonstration of the asymptotic efficiency of MMA in independent samples. Section 8 presents the results of two simulation experiments.

\section{Approximating models}

Let $\left\{y_{t}, \mathbf{x}_{t}: t=1, \ldots, n\right\}$ be a sample where $y_{t}$ is realvalued and $\mathbf{x}_{t}=\left(x_{1 t}, x_{2 t}, \ldots\right)^{\prime}$ is countably infinite. Consider the regression model

$y_{t}=\mu_{t}+e_{t}$

$\mu_{t}=\sum_{j=1}^{\infty} a_{j} x_{j t}=\mathbf{a}^{\prime} \mathbf{x}_{t}$

$E\left(e_{t} \mid \mathbf{x}_{t}\right)=0$

$E\left(e_{t}^{2} \mid \mathbf{x}_{t}\right)=\sigma^{2}$.

In Eq. (2), $\mathbf{a}=\left(a_{1}, a_{2}, \ldots\right)^{\prime}$ denotes a countably infinite vector, and we assume that sum (2) converges in mean square.
The goal is to construct a point forecast $f_{n+1}$ of $y_{n+1}$ given $\mathbf{x}_{n+1}$. The optimal mean-square forecast is the conditional mean $\mu_{n+1}$, and therefore empirical forecasts are estimates of $\mu_{n+1}$.

As (2) possibly contains an infinite number of coefficients but the sample size is finite, a finite-dimensional approximating model is estimated in practice. Suppose we have a set of $M$ approximating models where the $m$ 'th uses the first $k(m)$ regressors. (The leading case sets $k(m)=m$.) This imposes the strong assumption that the set of forecasting models is strictly nested. This is highly restrictive (as there are many cases where non-nested models are of interest) but is necessary for application of the methods discussed in this paper. Extending the methods to allow for non-nested models would be highly desirable.

Letting $\mathbf{x}_{t}(m)=\left(x_{1 t}, x_{2 t}, \ldots, x_{k(m) t}\right)^{\prime}$ and $\mathbf{a}(m)=\left(a_{1}, a_{2}\right.$, $\left.\ldots, a_{k(m)}\right)^{\prime}$ this model can be written as

$y_{t}=\mathbf{a}(m)^{\prime} \mathbf{x}_{t}(m)+e_{t}(m)$

where

$e_{t}(m)=e_{t}+\sum_{j=k(m)+1}^{\infty} a_{j} x_{j t}$,

or in matrix notation

$\mathbf{y}=\mathbf{X}(m) \mathbf{a}(m)+\mathbf{e}(m)$.

The least-squares estimate of $\mathbf{a}(m)$ is

$\hat{\mathbf{a}}(m)=\left(\mathbf{X}(m)^{\prime} \mathbf{X}(m)\right)^{-1} \mathbf{X}(m)^{\prime} \mathbf{y}$,

the least-squares residuals are

$\hat{\mathbf{e}}(m)=\mathbf{y}-\mathbf{X}(m) \hat{\mathbf{a}}(m)$,

and residual variance estimate

$\hat{\sigma}^{2}(m)=\frac{1}{n} \hat{\mathbf{e}}(m)^{\prime} \hat{\mathbf{e}}(m)$

The least-squares forecast of $y_{n+1}$ from this approximating model is then

$\hat{f}_{n+1}(m)=\mathbf{x}_{n+1}(m)^{\prime} \hat{\mathbf{a}}(m)$.

Some forecast combination methods require the calculation of recursive estimates. Stacking the first $t-1$ observations on $y_{t}$ and $\mathbf{x}_{t}(m)^{\prime}$ into the $(t-1) \times 1$ and $(t-1) \times k(m)$ matrices $\mathbf{y}_{t-1}$ and $\mathbf{X}_{t-1}(m)$, the recursive least-squares estimates of $\mathbf{a}(m)$ are

$\hat{\mathbf{a}}_{t-1}(m)=\left(\mathbf{X}_{t-1}(m)^{\prime} \mathbf{X}_{t-1}(m)\right)^{-1} \mathbf{X}_{t-1}(m)^{\prime} \mathbf{y}_{t-1}$.

The recursive least-squares forecasts of $y_{t}$ from this approximating model are then

$\hat{f}_{t}(m)=\mathbf{x}_{t}(m)^{\prime} \hat{\mathbf{a}}_{t-1}(m)$,

the forecast errors are

$\tilde{e}_{t}(m)=y_{t}-\hat{f}_{t}(m)$

and an estimate of the forecast error variance is

$\tilde{\sigma}^{2}(m)=\frac{1}{P+1} \sum_{t=n-P}^{n} \tilde{e}_{t}(m)^{2}$

for some integer $P$ such that $\tilde{e}_{n-P}(m)$ is well defined. 


\section{Forecast combination}

Section 2 defined a set of $M$ approximating models and associated forecasts. We now consider combinations of these forecasts. Let $w(m)$ be a weight assigned to the $m$ 'th forecast. Define the vectors $\mathbf{w}=(w(1), \ldots, w(M))$ and $\hat{\mathbf{f}}_{t}=$ $\left(\hat{f}_{t}(1), \hat{f}_{t}(2), \ldots, \hat{f}_{t}(M)\right)^{\prime}$. The combination forecast of $y_{n+1}$ is

$\hat{\mathbf{f}}_{n+1}(\mathbf{w})=\mathbf{w}^{\prime} \hat{\mathbf{f}}_{n+1}=\sum_{m=1}^{M} w(m) \hat{f}_{n+1}(m)=\mathbf{x}_{n+1}^{\prime} \hat{\mathbf{a}}(\mathbf{w})$

where

$\hat{\mathbf{a}}(\mathbf{w})=\sum_{m=1}^{M} w(m) \hat{\mathbf{a}}(m)$

is an averaging estimator of $\mathbf{a}$. In (8) and (9) we interpret $\mathbf{a}(\mathrm{m})$ and $\hat{\mathbf{a}}(\mathbf{w})$ to be infinite-dimensional vectors where $\hat{\mathbf{a}}(\mathrm{m})$ has entries of 0 beyond the $m$ 'th element.

Several methods have been proposed for selection of the weight vector $\mathbf{w}$. A classic method introduced by Bates and Granger (1969) sets the weights to be inversely proportional to the estimated forecast error variances defined in (7):

$w(m)=\frac{\tilde{\sigma}^{2}(m)^{-1}}{\sum_{j=1}^{M} \tilde{\sigma}^{2}(j)^{-1}}$.

This can be viewed as a smoothed version of predictive least squares (Rissanen, 1986), which simply selects the model with smallest forecast variance $\tilde{\sigma}^{2}(m)$.

Granger and Ramanathan (1984) proposed selecting the weights by minimizing the sum of squared forecast errors from the combination forecast

$Q(\mathbf{w})=\sum_{t=n-P}^{n}\left(y_{t}-\hat{\mathbf{f}}_{t}^{\prime} \mathbf{w}\right)^{2}$.

The unrestricted minimizer (Granger-Ramanathan's Method A) is the least-squares coefficient

$\hat{\mathbf{w}}=\left(\sum_{t=n-P}^{n} \hat{\mathbf{f}}_{t} \hat{\mathbf{f}}_{t}^{\prime}\right)^{-1} \sum_{t=n-P}^{n} \hat{\mathbf{f}}_{t} y_{t}$.

As described in Timmermann (2006, Sections 3.2 and 5.2) it may be prudent to minimize (11) subject to the convexity constraints $0 \leq w(m) \leq 1$ and additivity constraint $\sum_{m=1}^{M} w(m)=1$. This is

$\hat{\mathbf{w}}=\underset{0 \leq w(m) \leq 1, \sum_{m=1}^{M} w(m)=1}{\operatorname{argmin}} Q(\mathbf{w})$.

We call the forecast using (13) the constrained GrangerRamanathan forecast.

The recent forecasting literature has devoted considerable attention to forecasts based on Bayesian Model Averaging (BMA). When the priors are diffuse the BMA weights approximately equal

$w(m)=\frac{\exp \left(-\frac{1}{2} \operatorname{BIC}(m)\right)}{\sum_{j=1}^{M} \exp \left(-\frac{1}{2} \operatorname{BIC}(j)\right)}$

where

$\operatorname{BIC}(m)=n \ln \left(\hat{\sigma}^{2}(m)\right)+k(m) \ln n$ is the Bayesian Information Criterion (BIC) for model $m$. A related proposal (Buckland et al. (1997) and Burnham and Anderson (2002)) is smoothed AIC (SAIC) weights

$w(m)=\frac{\exp \left(-\frac{1}{2} \operatorname{AIC}(m)\right)}{\sum_{j=1}^{M} \exp \left(-\frac{1}{2} \operatorname{AIC}(j)\right)}$

where

$\operatorname{AIC}(m)=n \ln \left(\hat{\sigma}^{2}(m)\right)+2 k(m)$

is the Akaike Information Criterion (AIC).

The recent forecasting literature has also suggested the use of very simple combination methods, including the forecast mean which is equivalent to setting $w(m)=1 / M$, and the forecast median.

\section{Mallows Model Averaging}

In this paper we propose constructing forecast combinations using the weight vector selected by the Mallows Model Averaging (MMA) criterion of Hansen (2007), an extension of the classic Mallows criterion for model selection. The full-sample averaging estimator of the conditional mean $\mu_{t}$ is $\hat{\boldsymbol{\mu}}_{t}^{\prime} \mathbf{w}=\hat{\mathbf{a}}(\mathbf{w})^{\prime} \mathbf{x}_{t}$ where $\hat{\boldsymbol{\mu}}_{t}=\left(\hat{\mu}_{t}(1), \hat{\mu}_{t}(2), \ldots, \hat{\mu}_{t}(M)\right)^{\prime}$ and $\hat{\mu}_{t}(m)=\mathbf{x}_{t}(m)^{\prime} \hat{\mathbf{a}}(m)$. The MMA criterion is the penalized sum of squared residuals

$$
\begin{aligned}
C_{n}(\mathbf{w}) & =\sum_{t=1}^{n}\left(y_{t}-\hat{\boldsymbol{\mu}}_{t}^{\prime} \mathbf{w}\right)^{2}+2 \sum_{m=1}^{M} w(m) k(m) s^{2} \\
& =\mathbf{w}^{\prime} \hat{\mathbf{e}}^{\prime} \mathbf{e} \mathbf{w}+2 \mathbf{w}^{\prime} \mathbf{K} s^{2}
\end{aligned}
$$

where $\hat{\mathbf{e}}=[\hat{\mathbf{e}}(1), \ldots, \hat{\mathbf{e}}(M)], \mathbf{K}=(k(1), \ldots, k(M))^{\prime}$ and

$s^{2}=\frac{1}{n-k(M)} \hat{\mathbf{e}}(M)^{\prime} \hat{\mathbf{e}}(M)$

is an estimate of $\sigma^{2}$ from the largest fitted model. The second equality in (16) uses the assumption that the weights sum to one, which will be imposed.

The MMA weight vector is the value of $\mathbf{w}$ which minimizes $C_{n}(\mathbf{w})$. Feasible values for $\mathbf{w}$ are weight vectors whose elements are non-negative and sum to one. ${ }^{1}$ This set is the unit simplex in $\mathbb{R}^{M}$ :

$\mathscr{H}=\left\{\mathbf{w} \in[0,1]^{M}: \sum_{m=1}^{M} w^{m}=1\right\}$.

The definition of the Mallows weight vector is then

$\hat{\mathbf{w}}=\underset{\mathbf{w} \in \mathscr{H}}{\operatorname{argmin}} C_{n}(\mathbf{w})$.

The MMA forecasts are $\hat{\mu}_{t}^{\prime} \hat{\mathbf{w}}=\hat{\mathbf{a}}(\hat{\mathbf{w}})^{\prime} \mathbf{x}_{t}$. Due to the inequality constraints in (17), the solution to (18) is not analytically available for $M>3$, and must be found numerically using quadratic programming, for which numerical solutions have been thoroughly studied and algorithms are widely available. Even when $M$ is quite large the numerical solution to (18) is computationally fast using any standard algorithm.

\footnotetext{
${ }^{1}$ It is tempting to consider more weight vectors which do not satisfy these restrictions. However, if the Mallows criterion is used, we must restrict the weight vector to the unit simplex. If the non-negativity restriction is relaxed, the minimized values can be quite ill-behaved and have terrible empirical performance.
} 


\section{Mallows criterion and MSE}

A traditional motivation for the Mallows criterion is that it is an (approximately) unbiased estimate of the in-sample meansquared error. Hansen (2007) shows that this property holds for the criterion (16) for iid observations. In this section we develop an asymptotic analog for stationary dependent observations.

Write model (1) in vector notation as $\mathbf{y}=\boldsymbol{\mu}+\mathbf{e}$. The averaging estimator of $\boldsymbol{\mu}$ given the weight vector $\mathbf{w}$ is

$\hat{\boldsymbol{\mu}}(\mathbf{w})=\sum_{j=1}^{M} w(m) \mathbf{X}(m) \hat{\mathbf{a}}(m)=\mathbf{P}(\mathbf{w}) \mathbf{y}$

where

$\mathbf{P}(\mathbf{w})=\sum_{j=1}^{M} w(m) \mathbf{X}(m)\left(\mathbf{X}(m)^{\prime} \mathbf{X}(m)\right)^{-1} \mathbf{X}(m)^{\prime}$.

The averaging residual vector is $\hat{\mathbf{e}}(\mathbf{w})=\mathbf{y}-\hat{\boldsymbol{\mu}}(\mathbf{w})$.

Define the in-sample mean-squared error

$$
\begin{aligned}
L_{n}(\mathbf{w}) & =E\left(\frac{1}{n} \sum_{t=1}^{n}\left(\mu_{t}-\hat{\mu}_{t}(\mathbf{w})\right)^{2}\right) \\
& =\frac{1}{n} E(\boldsymbol{\mu}-\hat{\boldsymbol{\mu}}(\mathbf{w}))^{\prime}(\boldsymbol{\mu}-\hat{\boldsymbol{\mu}}(\mathbf{w})) .
\end{aligned}
$$

This is a summary measure of the fit of the averaging estimator.

Computing the sum of squared errors and expanding the square we find

$$
\begin{aligned}
\hat{\mathbf{e}}(\mathbf{w})^{\prime} \hat{\mathbf{e}}(\mathbf{w})= & (\mathbf{e}+\boldsymbol{\mu}-\hat{\boldsymbol{\mu}}(\mathbf{w}))^{\prime}(\mathbf{e}+\boldsymbol{\mu}-\hat{\boldsymbol{\mu}}(\mathbf{w})) \\
= & (\boldsymbol{\mu}-\hat{\boldsymbol{\mu}}(\mathbf{w}))^{\prime}(\boldsymbol{\mu}-\hat{\boldsymbol{\mu}}(\mathbf{w}))+\mathbf{e}^{\prime} \mathbf{e} \\
& +2 \mathbf{e}^{\prime}(\boldsymbol{\mu}-\hat{\boldsymbol{\mu}}(\mathbf{w})) \\
= & (\boldsymbol{\mu}-\hat{\boldsymbol{\mu}}(\mathbf{w}))^{\prime}(\boldsymbol{\mu}-\hat{\boldsymbol{\mu}}(\mathbf{w}))+\mathbf{e}^{\prime} \mathbf{e} \\
& +2 \mathbf{e}^{\prime}(\mathbf{I}-\mathbf{P}(\mathbf{w})) \boldsymbol{\mu}-2 \mathbf{e}^{\prime} \mathbf{P}(\mathbf{w}) \mathbf{e} .
\end{aligned}
$$

Thus the expectation for the Mallows Criterion is

$$
\begin{aligned}
& E\left(C_{n}(\mathbf{w})\right)=E\left(\hat{\mathbf{e}}(\mathbf{w})^{\prime} \hat{\mathbf{e}}(\mathbf{w})+2 \sum_{m=1}^{M} w(m) k(m) s^{2}\right) \\
& \simeq n\left(L_{n}(\mathbf{w})+\sigma^{2}\right)-2\left(E\left(\mathbf{e}^{\prime} \mathbf{P}(\mathbf{w}) \mathbf{e}\right)-\sigma^{2} \sum_{m=1}^{M} w(m) k(m)\right) .
\end{aligned}
$$

The final term is asymptotically zero, as we now show.

Theorem 1. If $\left(\mathbf{x}_{t}, e_{t}\right)$ is strictly stationary and ergodic, E $\mathbf{x}_{t}^{\prime} \mathbf{x}_{t}<\infty$, and $M$ and $\mathbf{w}$ are fixed as $n \rightarrow \infty$, then

$E\left(\mathbf{e}^{\prime} \mathbf{P}(\mathbf{w}) \mathbf{e}\right) \longrightarrow \sigma^{2} \sum_{m=1}^{M} w(m) k(m)$.

Using Theorem 1 , we see that $E\left(C_{n}(\mathbf{w})\right) \simeq n\left(L_{n}(\mathbf{w})+\sigma^{2}\right)$. Thus the expectation of the Mallows criterion is asymptotically equivalent to the squared error (19). (The additive and multiplicative constants do not matter.) We see that this classic property of the Mallows criterion extends to model averaging with stationary dependent data.

\section{MSE and MSFE}

In this section we show that the mean-squared forecast error (MSFE) approximately equals $L_{n}(\mathbf{w})$ when the observations are strictly stationary, and thus the Mallows criterion can also be seen as an approximately unbiased estimate of the MSFE. The one-stepahead out-of-sample forecast of $y_{n+1}$ given $\mathbf{x}_{n+1}$ is

$\hat{y}_{n+1}=\hat{\mathbf{a}}(\mathbf{w})^{\prime} \mathbf{x}_{n+1}$

so the one-step-ahead second-order MSFE is

$$
\begin{aligned}
R_{n}(\mathbf{w}) & =E\left(y_{n+1}-\hat{y}_{n+1}\right)^{2}-\sigma^{2} \\
& =E\left(e_{n+1}+(\mathbf{a}-\hat{\mathbf{a}}(\mathbf{w}))^{\prime} \mathbf{x}_{n+1}\right)^{2}-\sigma^{2} \\
& =E\left((\mathbf{a}-\hat{\mathbf{a}}(\mathbf{w}))^{\prime} \mathbf{x}_{n+1}\right)^{2} \\
& \simeq E\left((\mathbf{a}-\hat{\mathbf{a}}(\mathbf{w}))^{\prime} \mathbf{x}_{t}\right)^{2} \\
& =E\left(\mu_{t}-\hat{\mu}_{t}(\mathbf{w})\right)^{2} \\
& =L_{n}(\mathbf{w}) .
\end{aligned}
$$

The approximation in the fourth line is valid for stationary observations due to the approximate independence of $\mathbf{x}_{t}$ and $\hat{\mathbf{a}}(\mathbf{w})$ in large samples (and similarly for $\mathbf{x}_{n+1}$ and $\hat{\mathbf{a}}(\mathbf{w})$ ).

Combined with Theorem 1, we deduce that the Mallows criterion is an approximately unbiased estimate of the MSFE $R_{n}(\mathbf{w})$.

\section{Asymptotic efficiency of MMA}

The previous sections showed that the Mallows criterion is an asymptotically unbiased estimator of the MSE $L_{n}(\mathbf{w})$ and MSFE $R_{n}(\mathbf{w})$ for strictly stationary observations. This suggests that the weights which minimize the Mallows criterion may be asymptotically optimal with respect to this criterion. We have not been able to establish this result for dependent data, but building on the work of Li (1987) for efficient model selection procedures, Hansen (2007) established this asymptotic optimality property when the observations are independent. We report this result here for completeness.

For some finite integer $N$ let the weights $w(m)$ be restricted to the set $\left\{0, \frac{1}{N-1}, \frac{2}{N-1}, \ldots, 1\right\}$, let $\mathscr{H}^{*}$ be the subset of $\mathscr{H}$ restricted to this set of weights, and let $\hat{\mathbf{w}}^{*}$ be the MMA weights selected when restricted to $\mathscr{H}^{*}$ :

$\hat{\mathbf{w}}^{*}=\underset{\mathbf{w} \in \mathscr{H}^{*}}{\operatorname{argmin}} C_{n}(\mathbf{w})$.

Theorem 2. [ (Hansen, 2007)] If $\left(y_{t}, \mathbf{x}_{t}\right)$ are iid, satisfy (1)-(4),

$$
\begin{aligned}
& \sup _{t} E\left(e_{t}^{4 N} \mid \mathbf{x}_{t}\right)<\infty, \\
& \inf _{\boldsymbol{w} \in \mathscr{H}} E\left((\boldsymbol{\mu}-\hat{\boldsymbol{\mu}}(\mathbf{w}))^{\prime}(\boldsymbol{\mu}-\hat{\boldsymbol{\mu}}(\mathbf{w})) \mid \mathbf{x}_{1}, \ldots, \mathbf{x}_{n}\right) \rightarrow \infty,
\end{aligned}
$$

and $k(M) / n \rightarrow 0$, then the MMA weights $\hat{\mathbf{w}}^{*}$ are optimal in the sense that

$$
\frac{L_{n}\left(\hat{\mathbf{w}}^{*}\right)}{\inf _{w \in \mathscr{H}^{*}} L_{n}(\mathbf{w})} \rightarrow_{p} 1
$$

as $n \rightarrow \infty$. 
Theorem 2 shows that MMA is asymptotically efficient in the sense that its in-sample MSE is equivalent to the infeasible optimal averaging estimator. The assumptions are fairly minimal. Assumption (A.1) is a strong moment bound but may be a technical artifact of the proof method. The restriction of $\mathcal{H}$ to $\mathcal{H}^{*}$ also appears to be a technical artifact of the proof method. ${ }^{2}$ However, Assumption (A.2) is important. It states that there is no finite $m$ for which $\mu_{t}(m)$ equals $\mu_{t}$-equivalently, that all finite-dimensional models are approximations. This is an important condition for it is well known that when the true model is finite dimensional, then this class of model selection procedures is asymptotically inefficient.

Theorem 2 excludes dependent data and is therefore not directly relevant for time-series forecasting. However, there is a closely related theory of optimal model selection for one-stepahead forecasting in the context of autoregressive models, and we expect that the optimality property should carry over to forecast combination. The most relevant contribution is by Ing and Wei (2005) who consider stationary infinite-order autoregressions with iid innovations. Let $\hat{y}_{n+1}(m)$ be the one-step-ahead forecast of $y_{n+1}$ using the least-squares estimate of an autoregressive (AR) model of order $m$ and let $R_{n}(m)$ denote the second-order meansquared forecast error from these estimates:

$R_{n}(m)=E\left(y_{n+1}-\hat{y}_{n+1}(m)\right)^{2}-\sigma^{2}$.

Let $\hat{m}$ be the AR order selected by the Akaike or Mallows criterion. Ing and Wei (2005) show that $\hat{m}$ is asymptotically optimal in the sense that

$$
\frac{R_{n}(\hat{m})}{\inf _{1 \leq m \leq M} R_{n}(m)} \rightarrow p 1 .
$$

Model selection is a restricted version of model averaging. Given the similarity with Theorem 2 we conjecture that the optimality result (20) will extend to the case of general weight vectors.

However, the technical challenges for establishing this result are formidable. It is necessary to extend Lemmas 1,2 and 3 of Ing and Wei (2005) to the case of averaging estimators. (Ideally the results would resemble Theorem 2 of Whittle (1960).) Such an extension is beyond the scope of the present paper and is left for future research.

\section{Finite sample investigation}

We now investigate the finite sample MSFE of the our model average estimator in two simulation designs. The first is the regression model

$y_{t}=\theta_{0}+\sum_{k=1}^{K} \theta_{k} x_{k t}+e_{t}$.

The error $e_{t} \sim N\left(0, \sigma^{2}\right)$ is independent of the regressors $x_{k t}$, which are independent $\mathrm{AR}(1)$ processes $x_{k t}=\rho x_{k t-1}+u_{k t}$ where $\rho=0.5$ and $u_{k t} \sim N(0,0.75)$. We normalize $\sigma^{2}=1$ and set the regression coefficients by the rule

$\theta_{k}=c \gamma_{k}$
$\gamma_{k}=\frac{k^{\alpha} \beta^{k}}{\sum_{j=1}^{K} j^{2 \alpha} \beta^{2 j}}$.

\footnotetext{
2 It is highly desirable, but technically quite challenging, to relax this restriction. It is important to emphasize that this restriction is only relevant for the optimality theory, not for empirical application of the method.
}

By varying $\alpha$ and $\beta$ a variety of patterns for $\theta_{k}$ as a function of $k$ can be determined. Eight representative patterns were selected, and the coefficients $\gamma_{k}$ are displayed in the left panels of Figs. 1 and 2. In the first six panels the coefficients $\gamma_{k}$ are monotonically decreasing in $k$, as would be expected if the regressors were correctly ordered. The final two panels display cases of non-monotonic coefficients.

The parameter $c$ was set by the rule $c=\sqrt{R^{2} /\left(1-R^{2}\right)}$ and $R^{2}$ varied on a grid from 0.1 to 0.9 , as this is the population $R^{2}$ of this regression. We set the sample size to $n=200$ and set $K=12$. Forecasts of $y_{n+1}$ given $x_{n+1}$ are based on the linear regressions:

$y_{t}=\hat{\theta}_{0}+\sum_{k=1}^{m} \hat{\theta}_{k} x_{k t}+\hat{e}_{t}(m)$

for $m=0, \ldots, 12$, estimated by OLS. ${ }^{3}$

The second design is the moving average model

$y_{t}=\sum_{k=0}^{\infty} \theta_{k} e_{t-k}$

where $e_{t}$ is iid $N\left(0, \sigma^{2}\right)$ and $n=200$. We normalize $\sigma^{2}=1$ and set the coefficients using the rule

$\theta_{k}=(1+k)^{\alpha} \beta^{k}$.

By varying $\alpha$ and $\beta$ a variety of moving average patterns can be generated. We varied $\alpha$ among $\{0,0.25,0.50$ and 1.0$\}$ and varied $\beta$ on a grid from .6 to .9 . The moving average coefficient patterns are displayed in the four left panels of Fig. 3.

Forecasts of $y_{n+1}$ are based on $\operatorname{AR}(\mathrm{m})$ models:

$y_{t}=\hat{\mu}+\hat{\alpha}_{1} y_{t-1}+\cdots+\hat{\alpha}_{m} y_{t-m}+\hat{e}_{t}(m)$

for $m=0, \ldots, 12$, estimated by OLS.

In both experiments a wide set of forecast combination methods were compared, including AIC selection, smoothed AIC (SAIC) (15), BIC selection, weighted BIC (WBIC) (14), PLS selection, median forecast, mean forecast, Bates-Granger combination (10), Granger-Ramanathan combination (12), Constrained Granger-Ramanathan combination (13), and MMA combination (18).

We compare the forecasting methods based on out-of-sample second-order mean-square forecast error (MSFE)

$\operatorname{MSFE}=\frac{n}{\sigma^{2}}\left(E\left(y_{n+1}-\hat{y}_{n+1}\right)^{2}-\sigma^{2}\right)$.

The error variance $\sigma^{2}$ is subtracted because it is the leading term in the MSFE, and is common across forecast methods. The scaling $\left(n / \sigma^{2}\right)$ is used to render the results scale-free. We compute MSFE by computing averages across 20,000 simulation draws.

Comparing the forecast methods across the parameter settings, we find that eight of the methods are dominated (either uniformly or nearly so) by one of the three other methods. Specifically, BIC selection is dominated by WBIC, AIC by SAIC and SAIC by MMA. The mean and median forecasts are dominated by the Bates-Granger combination. Granger-Ramanathan is strongly dominated by constrained Granger-Ramanathan and PLS, which are in turn dominated by MMA. To keep our graphs uncluttered, only the three undominated methods (WBIC, Bates-Granger, and MMA) are displayed.

For each experiment, MSFE is displayed in the right panels of Figs. 1-3. (The left panels display the coefficient pattern.) Figs. 1 and 2 display results for the first simulation design (the regression model) and Fig. 3 displays results for the second design (the

\footnotetext{
${ }^{3}$ To assess robustness to misspecification, the simulation was also done with the regression models restricted to $m=0, \ldots, 6$. The results were qualitatively unchanged.
} 

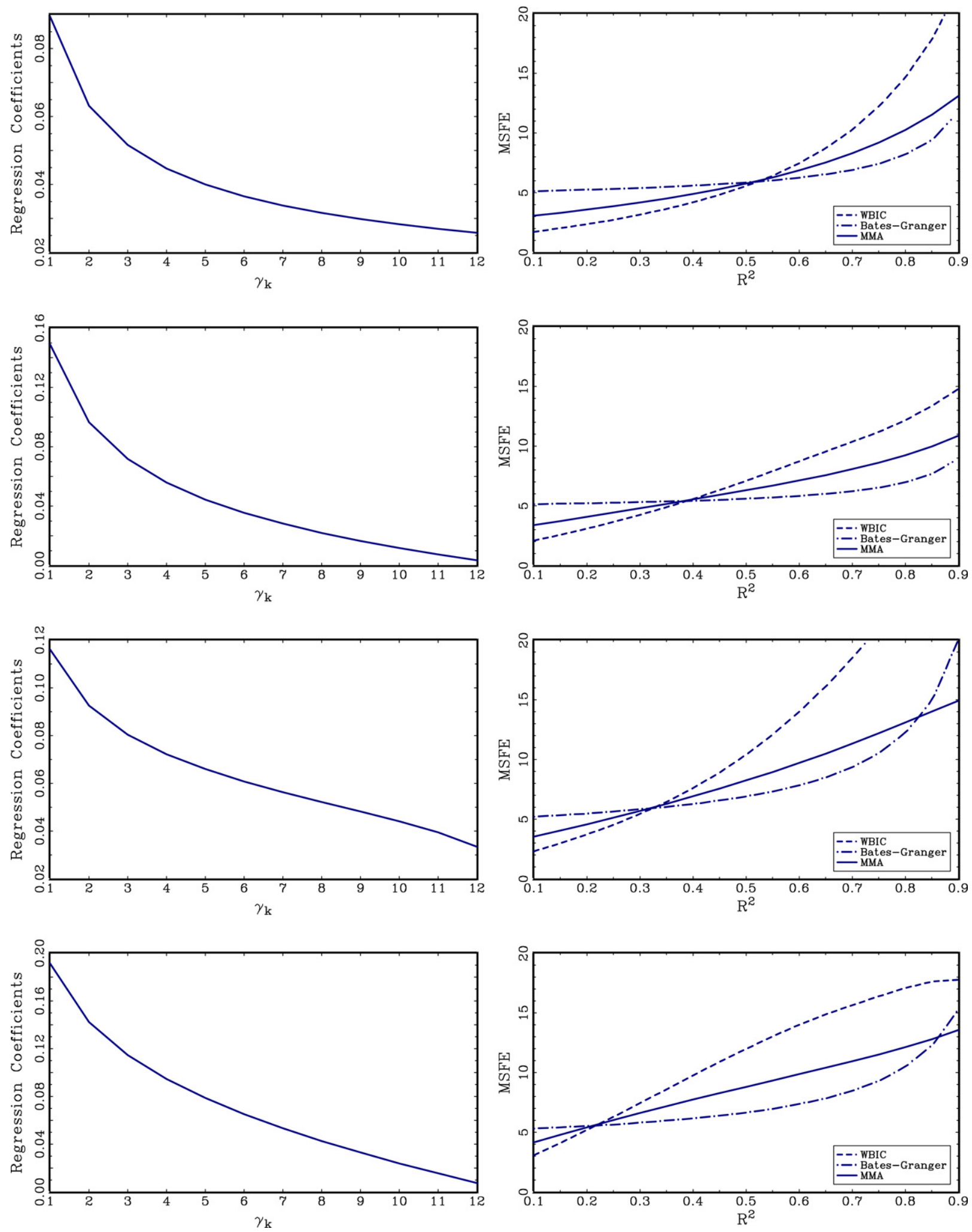

Fig. 1. Regression model, cases 1-4.

moving average model). In Figs. 1 and 2 the coefficient pattern $\gamma_{k}$ is displayed in the left panel, and the right panel displays MSFE as $R^{2}$ is varied from 0.1 to 0.9 . In Fig. 3 the coefficient $\alpha$ is held fixed at one of the values $\{0,0.25,0.50$ and 1.0$\}$, and the right panel displays MSFE as $\beta$ is varied from 0.6 to 0.9 .
In all plots, the MSFE of WBIC forecasts is displayed with the dashed line, the MSFE of Bates-Granger forecasts with the dashdotted line, and the MSFE of MMA forecasts with the solid line. Examining the twelve panels in the three figures, there is no one method which uniformly dominates the others. 

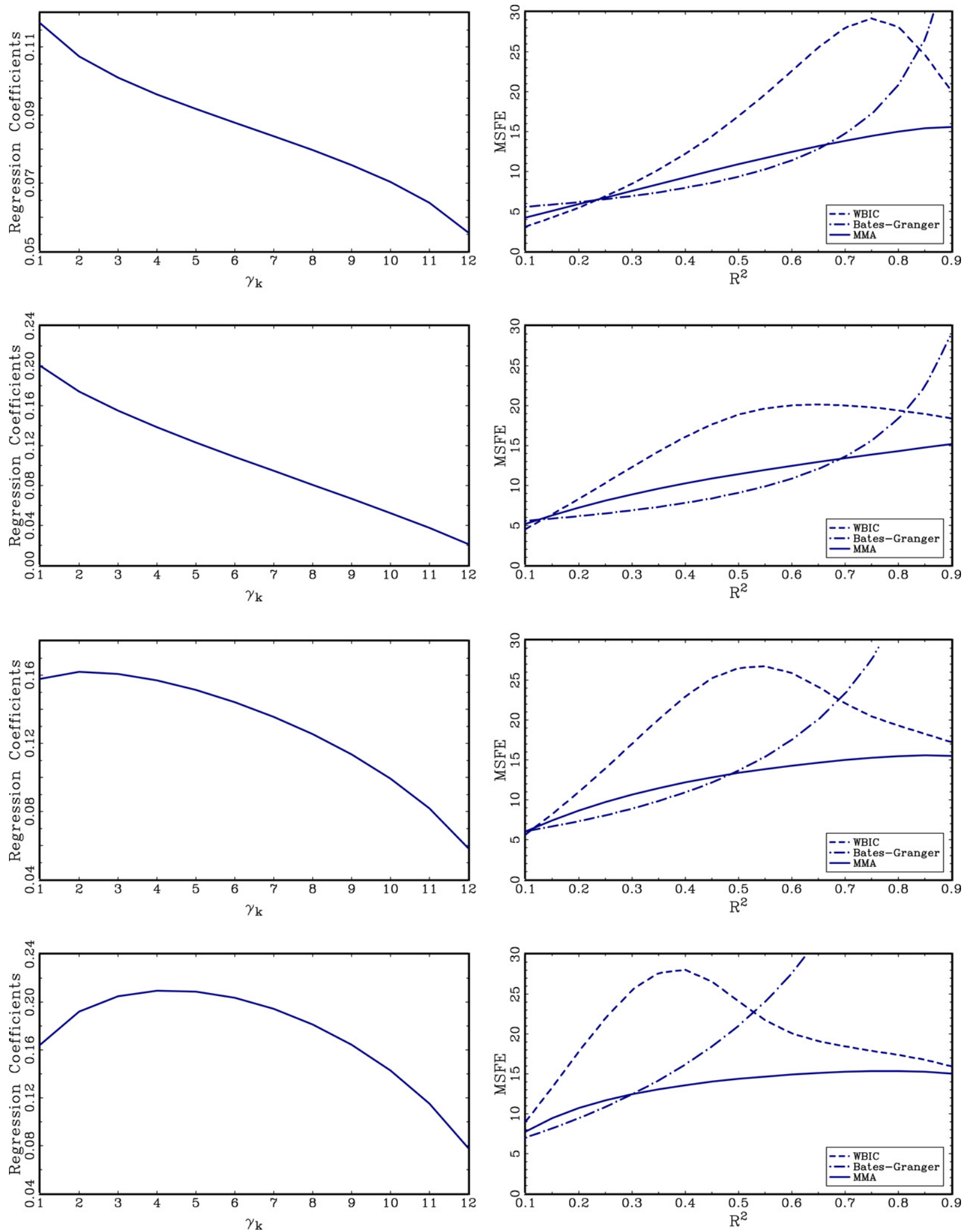

Fig. 2. Regression model, cases 5-8.

One criterion to compare procedures is maximum regret. For a given parameterization the regret of a procedure is the difference between its risk (MSFE) and the best achievable risk-in this case the best MSFE among the eleven forecasting methods. The maximum regret of a procedure is the maximum value of this regret across all parameterizations. If a procedure has low regret it means that for all parameterizations the risk of that procedure is not too far from the best achievable MSFE. We calculated the maximum regret of the forecasting methods for each simulation design and report the results in Table 1, roughly ordered from worst performance (highest regret) to best performance (lowest regret).

Table 1 shows that the procedures have dramatically different performance as measured by regret. By a wide margin the 

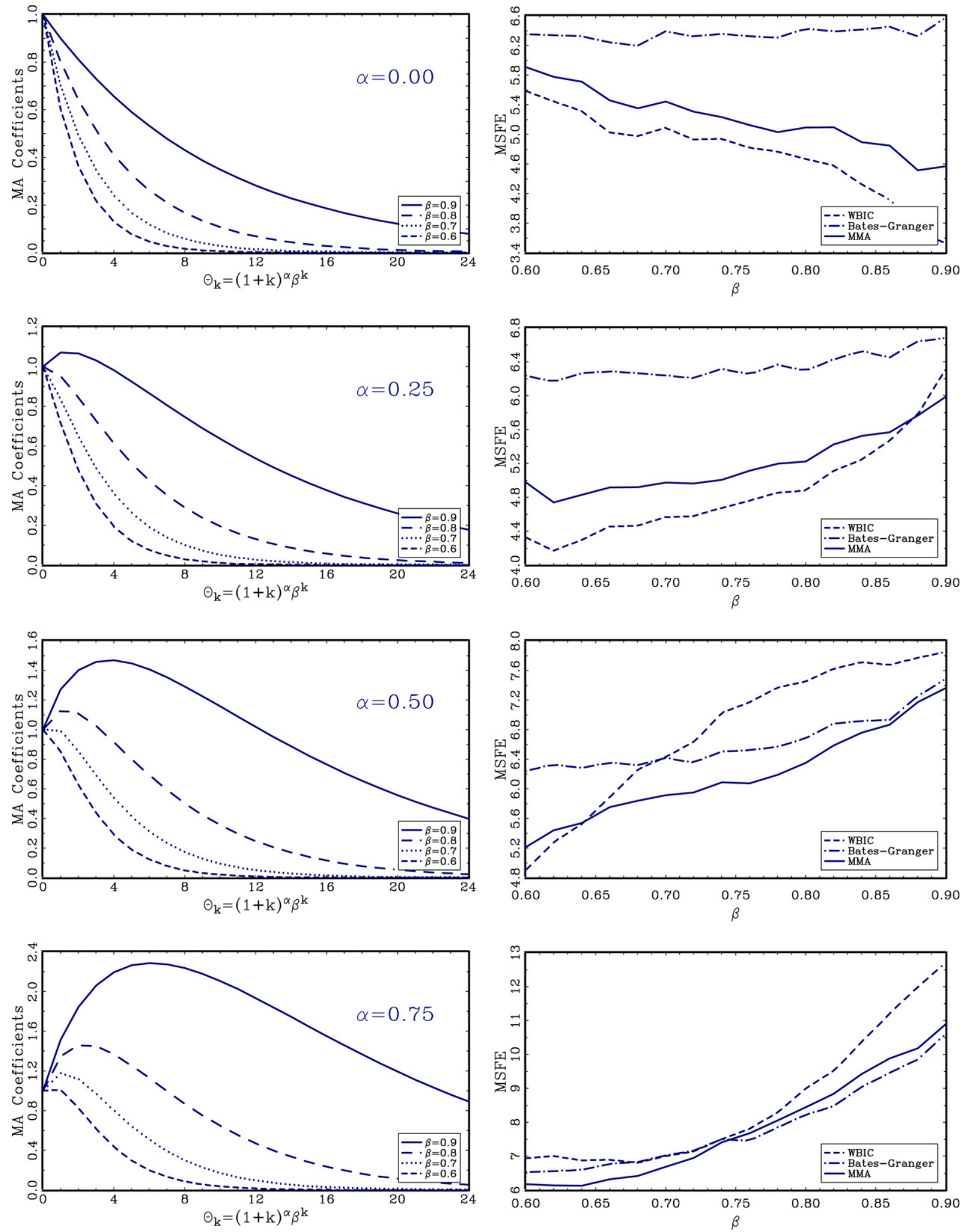

Fig. 3. Moving average model.

procedure with the best performance is MMA, with SAIC and constrained Granger-Ramanathan closely behind. What the results show (and this is consistent with a close scrutiny of Figs. 1-3) is that for all parameterizations the MMA forecast has a MSFE which is either the best, or reasonably close to the best. Other procedures (such as WBIC and Bates-Granger) may perform quite well for certain parameterizations, but there are parameterizations for which these procedures have quite large MSFE relative to other estimators.

The simulation exercises confirm our expectations. Forecast combination generally achieves lower MSFE than forecast selection. A feasible and easy-to-apply method with particularly low 
Table 1

Maximum regret

\begin{tabular}{lcc}
\hline & Design 1 & Design 2 \\
\hline Mean & 146.0 & 100.0 \\
Median & 145.0 & 3.3 \\
Granger-Ramanathan & 37.1 & 31.2 \\
Bates-Granger & 70.0 & 3.0 \\
BIC & 20.1 & 4.1 \\
WBIC & 14.8 & 2.7 \\
Predictive least squares & 8.0 & 3.5 \\
AIC & 6.8 & 2.7 \\
Constrained Granger-Ramanathan & 4.0 & 3.3 \\
SAIC & 3.9 & 1.4 \\
Mallows Model Averaging & 2.6 & 1.0 \\
\hline
\end{tabular}

MSFE for one-step-ahead forecasting is Mallows Model Averaging. In our simulations, it performed uniformly well across a range of models and parameterizations, yielding quite low regret, meaning that it is hard to do better than this method.

\section{Acknowledgements}

This research was supported by the National Science Foundation. The author thanks Ken West, Tim Cogley and two referees for helpful comments and suggestions on an earlier draft.

\section{Appendix}

Proof of Theorem 1. For any $m$, by the ergodic theory and the central limit theorem for martingale difference sequences,

$\frac{1}{n} \mathbf{X}(m)^{\prime} \mathbf{X}(m) \stackrel{p}{\longrightarrow} V(m)=E\left(\mathbf{x}_{t}(m) \mathbf{X}_{t}(m)^{\prime}\right)$

and

$\frac{1}{\sqrt{n}} \mathbf{X}(m)^{\prime} \mathbf{e} \stackrel{d}{\longrightarrow} N\left(0, V(m) \sigma^{2}\right)$.

Therefore

$\mathbf{e}^{\prime} \mathbf{X}(m)\left(\mathbf{X}(m)^{\prime} \mathbf{X}(m)\right)^{-1} \mathbf{X}(m)^{\prime} \mathbf{e} \stackrel{d}{\longrightarrow} \sigma^{2} \chi_{k(m)}^{2}$

where $\chi_{k(m)}^{2}$ is chi-square with $k(m)$ degrees of freedom, and

$E\left(\mathbf{e}^{\prime} \mathbf{X}(m)\left(\mathbf{X}(m)^{\prime} \mathbf{X}(m)\right)^{-1} \mathbf{X}(m)^{\prime} \mathbf{e}\right) \longrightarrow \sigma^{2} k(m)$.

It follows that for any fixed $M$ and $\mathbf{w}$,

$$
\begin{aligned}
E\left(\mathbf{e}^{\prime} \mathbf{P}(\mathbf{w}) \mathbf{e}\right) & =\sum_{j=1}^{M} w(m) E\left(\mathbf{e}^{\prime} \mathbf{X}(m)\left(\mathbf{X}(m)^{\prime} \mathbf{X}(m)\right)^{-1} \mathbf{X}(m)^{\prime} \mathbf{e}\right) \\
& \longrightarrow \sigma^{2} \sum_{j=1}^{M} w(m) k(m)
\end{aligned}
$$

as claimed.

\section{References}

Akaike, H., 1973. Information theory and an extension of the maximum likelihood principle. In: Petroc, B., Csake, F. (Eds.), Second International Symposium on Information Theory.

Akaike, H., 1979. A Bayesian extension of the minimum AIC procedure of autoregressive model fitting. Biometrika 66, 237-242.

Avramov, D., 2002. Stock return predictability and model uncertainty. Journal of Finance 64, 423-458.

Banasali, R.J., 1996. Asymptotically efficient autoregressive model selection for multistep prediction. Annals of the Institute of Statistical Mathematics 48, 577-602.

Bates, J.M., Granger, C.M.W., 1969. The combination of forecasts. Operations Research Quarterly 20, 451-468.
Brock, William, Durlauf, Stephen, 2001. Growth empirics and reality. World Bank Economic Review 15, 229-272.

Brock, William, Durlauf, Stephen, West, Kenneth D., 2003. Policy analysis in uncertain economic environments. Brookings Papers on Economic Activity 1 235-322.

Buckland, S.T., Burnhamn, K.P., Augustin, N.H., 1997. Model selection: An integral part of inference. Biometrics 53, 603-618.

Burnham, Kenneth P., Anderson, David R., 2002. Model Selection and Multimodel Inference: A Practical Information-Theoretic Approach, Second ed.. Springer, New York.

Clemen, R.T., 1989. Combining forecasts: A review and annotated bibliography. International Journal of Forecasting 5, 559-581.

Diebold, F.X., Lopez, J.A., 1996. Forecast evaluation and combination. In: Maddala, Rao (Eds.), Handbook of Statistics. Elsevier, Amsterdam.

Fernandez, Ley, C.E., Steel, M.F.J., 2001a. Benchmark priors for Bayesian model averaging. Journal of Econometrics 100, 381-427.

Fernandez, Ley, C.E., Steel, M.F.J., 2001b. Model uncertainty in cross-country growth regressions. Journal of Applied Econometrics 16, 563-576.

Garratt, A., Lee, K., Pesaran, M.H., Shin, Y., 2003. Forecasting uncertainties in macroeconomic modelling: An application to the UK economy. Journal of the American Statistical Association 98, 829-838.

Granger, C.W.J., 1989. Combining Forecasts-Twenty years later. Journal of Forecasting 8, 167-173.

Granger, C.W.J., Ramanathan, R., 1984. Improved methods of combining forecast accuracy. Journal of Forecasting 19, 197-204.

Hansen, Bruce E., 2007. Least squares model averaging. Econometrica 75, $1175-1189$.

Hendry, D.F., Clements, M.P., 2002. Pooling of forecasts. Econometrics Journal 5. 1-26.

Hjort, Nils Lid, Claeskens, Gerda, 2003. Frequentist model average estimators Journal of the American Statistical Association 98, 879-899.

Hoeting, Jennifer A., Madigan, David, Raftery, Adrian E., Volinsky, Chris T., 1999. Bayesian model averaging: A tutorial. Statistical Science 14, 382-417.

Ing, Ching-Kang, 2003. Multistep prediction in autoregressive processes. Econometric Theory $19,254-279$.

Ing, Ching-Kang, 2004. Selecting optimal multistep predictors for autoregressive processes of unknown order. Annals of Statistics 32, 693-722.

Ing, Ching-Kang, 2007. Accumulated prediction errors, information criteria and optimal forecasting for autoregressive time series. Annals of Statistics 35 1238-1277.

Ing, Ching-Kang, Wei, Ching-Zong, 2003. On same-realization prediction in an infinite-order autoregressive process. Journal of Multivariate Analysis 85, $130-155$.

Ing, Ching-Kang, Wei, Ching-Zong, 2005. Order selection for same-realization predictions in autoregressive processes. Annals of Statistics 33, 2423-2474.

Lee, Sangyeol, Karagrigoriou, Alex, 2001. An asymptotically optimal selection of the order of a linear process. Sankhya Series A 63, 93-106.

$\mathrm{Li}$, Ker-Chau, 1987. Asymptotic optimality for $C_{p}, C_{L}$, cross-validation and generalized cross-validation: Discrete Index Set. Annals of Statistics 15, 958-975.

Mallows, C.L., 1973. Some comments on $C_{p}$. Technometrics 15, 661-675.

Min, C.-K., Zellner, A., 1993. Bayesian and non-Bayesian methods for combining models and forecasts with applications to forecasting international growth rates. Journal of Econometrics 56, 89-118.

Rissanen, J., 1986. Order estimation by accumulated prediction errors. Journal of Applied Probability 23A, 55-61.

Sala-i-Martin, Xavier, Doppelhofer, Gernot, Miller, Ronald I., 2004. Determinants of long-term growth: A Bayesian averaging of classical estimates (BACE) approach. American Economic Review 94, 813-835.

Schwarz, G., 1978. Estimating the dimension of a model. Annals of Statistics 6 , 461-464.

Shibata, Ritaei, 1980. Asymptotically efficient selection of the order of the model for estimating parameters of a linear process. Annals of Statistics 8, 147-164.

Shibata, Ritaei, 1981. An optimal selection of regression variables. Biometrika 68 45-54.

Shibata, Ritaei, 1983. Asymptotic mean efficiency of a selection of regression variables. Annals of the Institute of Statistical Mathematics 35, 415-423.

Stock, J.H., Watson, M.W., 1999. A comparison of linear and nonlinear univariate models for forecasting macroeconomic time series. In: Engle, R., White, H.(Eds.), Cointegration, Causality and Forecasting: A Festschrift for Clive W.J. Granger. Oxford University Press.

Stock, J.H., Watson, M.W., 2004. Combination forecasts of output growth in a sevencountry data set. Journal of Forecasting 23, 405-430.

Stock, J.H., Watson, M.W., 2005. An empirical comparison of methods for forecasting using many predictors. Working paper, NBER.

Stock, J.H., Watson, M.W., 2006. Forecasting with many predictors. In: Elliott, G., Granger, C.W.J., Timmermann, A. (Eds.), Handbook of Economic Forecasting, vol. 1. Elsevier, Amsterdam, pp. 515-554.

Timmermann, Allan, 2006. Forecast combinations. In: Elliott, G., Granger, C.W.J. Timmermann, A. (Eds.), Handbook of Economic Forecasting, vol. 1. Elsevier, Amsterdam, pp. 135-196.

Whittle, P., 1960. Bounds for the moments of linear and quadratic forms in independent variables. Theory of Probability and Its Applications 5, 302-305.

Wright, Jonathan H., 2003a. Bayesian model averaging and exchange rate forecasting. Federal Reserve Board International Finance Discussion Papers 779.

Wright, Jonathan H., 2003b. Forecasting US Inflation by Bayesian Model Averaging. Federal Reserve Board International Finance Discussion Papers, 780. 